\title{
LISTA TAXONOMICA DE LAS ESPECIES DE BIVALVOS DULCEACUICOLAS EN EL PERU
}

\author{
KARINA VALDIVIA P. ${ }^{2,4}$ \\ RONALD VIVAR G. ${ }^{1,2}$ \\ PEDRO HUAMÁN M. ${ }^{1,4}$ \\ JAVIER ZALDÍVAR R. ${ }^{3}$
}
1. Departamento de Malacología y Carcinología. Museo Historia Natural Apdo. 14034, Lima 14. Perú 2. Laboratorio de Fauna Dulceacuícola. Instituto de Investigación «Antonio Raimondi». Fac. Ciencias Biológicas. Universidad Nac. Mayor de San Marcos. Apdo. 10235. 3. IVITA. Daniel A. Carrión 319. Pucallpa. 4. Laboratorio de Invertebrados. Fac. Ciencias Biológicas. Universidad Ricardo Palma. Av. Benavides cda. $54 \quad$ s/n. Fax. 449-2477. Lima.

\begin{abstract}
RESUMEN
Se presenta una lista taxonómica preliminar de 26 especies de bivalvos de agua dulce pertenecientes a 18 géneros y 5 familias de bivalvos de agua dulce.
\end{abstract}

PALABRAS CLAVE: Bivalvia, dulceacuícola, Perú.

\section{SUMMARY}

Introducing a preliminar taxonomical checklist of 26 species respected to 18 genera and 5 families from freshwater bivalves. KEY WORDS: Bivalvia, freshwater, Peru.

\section{INTRODUCCION}

Los bivalvos de ambientes límnicos en el Perú se encuentran distribuídos en la zona andina y selvática; presentan dificultades en su identificación por la variabilidad de sus valvas; producida por la ocupación de una diversidad de ambientes ecológicos debido a la compleja distribución de las cuencas hidrográficas en los ambientes continentales.

El conocimiento de las especies de bivalvos peruanos es escaso; las pocas contribuciones se basan en patrones descriptivos de las valvas. Un análisis detallado de algunos sistemas de órganos conducen a una mejor caracterización de las especies (Veitenheimer y Mansur, 1978; Mansur y Oliveira, 1990).

Examinamos material colectado durante algunos meses entre los años de 1991 a 1995, igualmente hemos considerado diferentes especies peruanas señaladas en algunos artículos sudamericanos (Bonetto,1965; Mansur y Valer, 1992).

En la presente comunicación, la composición taxonómica de los bivalvos amazónicos y altoandinos se agrupan en 5 familias, 18 géneros y 26 especies, que a continuación se detallan:

\section{FAMILIA ETHERIIDAE}

Bartlettia stefanensis (Moricand, 1856)

\section{FAMILIA HYRIIDAE}

Castalia ambigua Lamarck, 1819

Castalia schombergiana Sowerby, 1869

Diplodon obsolescens Baker, 1914

Diplodon suavidicus (Lea, 1856)

Callonaia Simpson, 1900

Triplodon corrugatus(Lamarck, 1819)

Prisodon obliquus Schumacher, 1817

Paxyodon syrmatophorus Meuschen, 1781

\section{FAMILIA MYCETOPODIDAE}

Anodontites elongatus (Swainson, 1823)

A. trapezeus (Spix, 1827)

A. trapesialis (Lamarck, 1819)

A. trigonus (Spix, 1827)

A. ensiformis (Spix , 1827)

A. schomburgianus (Sowerby, 1870)

Mycetopoda legumen (Martens, 1888)

M. siliquosa Spix, 1827

M. soleniformis Orbigny, 1835

Mycetopodella falcata (Higgins, 1868)

Tamsiella Hass, 1931

Iheringiella Pilsbryi, 1893

Leyla blainvilliana (Lea,1834)

L. esula (Orbigny, 1835)

Monocondylaea Orbigny, 1835 


\section{FAMILIA SPHAERIIDAE}

Sphaerium titicacense Pilsbry, 1924

S. lauricochae Philippi, 1869

S. forbesi Philippi, 1869

Pisidium meierbrooki Kuiper y Hinz,1983

Eupera simoni Jousseaume, 1889

\section{FAMILIA CORBICULIDAE}

\section{Corbicula sp.}

El presente trabajo constituye un aporte para el conocimiento de la diversidad malacológica; del total de especies (26), una parte ha sido revisada en aspectos anatómicos para caracterizar a los géneros y especies; las restantes son aportes de bibliografía sudamericana y de consultas con especialistas en la materia. Entre los grupos mencionados, algunas especies y géneros no han sido revisados desde el punto de vista anatómico o no se ha efectuado una revisión histórica del grupo en mención, quedando algunas dudas en la nomenclatura que permiten estimar alguna aparición de sinónimos intraespecíficos o intergenéricos en la familia Mycetopodidae, colocando en duda la validez del epíteto específico y en algunos casos el nombre en grupo del género.

Un análisis con mayor detalle en diferentes poblaciones de especies de bivalvos nos permitirá interpretar la variabilidad morfológica y las formas intermedias que ocasionalmente se producen y probablemente podemos confirmar las 24 entidades específicas o reducir el taxón específico por probable sinonimia.

El conocimiento de patrones de distribución de las especies permitirá conocer las áreas de diversificación y especiación utilizando el criterio de mayor diversidad y endemismo. Un muestreo de mayor intensidad en zonas andinas Nor -Orientales y en la cuenca amazónica nos permitirá obtener un inventario completo de nuestra malacotaxa de importancia económica con fines de conservación.

\section{AGRADECIMIENTOS}

El presente estudio fué subvencionado en parte por el Fondo Especial de Desarrollo Universitario (FEDU) (UNMSM) al proyecto $\mathrm{N}^{\circ}$ 4100127. A la Dra. Maria Cristina D. Mansur, de la Fundacão Zoobotánica do Rio Grande do Sul (Brasil), por el apoyo bibliográfico.

\section{REFERENCIAS BIBLIOGRAFICAS.}

BONETTO, A.A. 1965. Almejas Sudamericanas de la Tribu Castaliini. Physis 25(69): 187-196.

MANSUR, M.C. \& OLIVEIRA DA SILVA, M. 1990. Morfologia e microanatomia comparada de Bartlettia stefanensis (Moricand, 1856) e Anodontites tenebricosus (Lea, 1834) (Bivalvia, Unionoida, Muteloidea). Amazoniana 11 (2): 147 - 166.

MANSUR, M.C. \& VALER, R.M. 1992. Moluscos bivalves do Rio Uraricoera e Rio Branco, Roraima , Brasil. Amazoniana 12 (1): 85-100.

ORTMANN, A.E. 1921. South american naiades: A contribution to the knowledge of the freshwater mussels of South America. Memoirs of the Carnegie Museum, Pittsburg 8 (3): 451 - 686.

VEITENHEIMER, I. \& MANSUR , M.C. 1978. Morfologia, histologia e ecologia de Mycetopoda legumen (Martens, 1888) (Bivalvia, Mycetopodidae). Iheringia Ser. Zool. 52: 33-71. 